\title{
RATIONAL COHOMOLOGY OPERATIONS AND MASSEY PRODUCTS
}

\section{DAVID KRAINES}

Let $\boldsymbol{Q}$ be the group of rational numbers. Then $H^{*}(\boldsymbol{Q}, n ; \boldsymbol{Q})$ is either an exterior algebra or polynomial algebra on a class $u$ of dimension $n$. By the Künneth formula, if $P=\times_{j=1}^{s} K\left(Q, n_{j}\right)$, that is if $P$ is a rational generalized Eilenberg-MacLane space (GEM), then every class in $H^{k}(P ; Q)$ is a polynomial on the fundamental classes $\left\{u_{j}\right\}$. Thus every rational primary cohomology operation on $\left(x_{1}\right.$, $\left.\cdots, x_{s}\right)$ can be written $\Phi\left\{x_{j}\right\}=\sum \lambda_{j} x_{j}+\sum y_{r} z_{r}$ when $\lambda_{j} \in Q$ and $y_{r}, z_{r} \in \widetilde{H}^{*}(P ; Q)$. The decomposable term is the twofold matrix Massey product

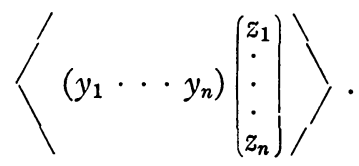

In this paper we show that rational higher order cohomology operations can be expressed as a linear term plus a sum of matrix Massey products. As a corollary we conclude that the only stable rational cohomology operations are addition and scalar multiplication.

In defining a rational cohomology operation we recall the notion of a rational Postnikov tower. Let $P_{0}=\times_{j=1}^{s} K\left(Q, n_{j}\right)$ where $n_{1}, \cdots$, $n_{s}$ are not necessarily distinct positive integers. We say that

$$
\begin{aligned}
& P_{m} \\
& \downarrow \pi_{m} \\
& P_{m-1} \stackrel{k_{m}}{\rightarrow} K\left(\boldsymbol{Q}, j_{m}\right) \\
& \downarrow \\
& \vdots \\
& \\
\downarrow & \pi_{1} \\
& P_{0} \stackrel{k_{1}}{\rightarrow} K\left(Q, j_{1}\right)
\end{aligned}
$$

is an $m+1$ stage rational Postnikov tower if $1<j_{1} \leq \cdots \leq j_{m}$ and $P_{r \rightarrow}^{{ }^{\pi} r} \rightarrow P_{r-1}$ is the fibration induced from the path loop fibration over $K\left(\boldsymbol{Q}, j_{r}\right)$ by the map $k_{r}$.

Received by the editors September 20, 1968. 
For $\odot$ a Postnikov tower as above, set $u_{j}=\pi^{*} u_{j}^{\prime}, j=1, \cdots, s$, where $u_{j}^{\prime} \in H^{n_{j}}\left(P_{0} ; Q\right)$ is the $j$ th fundamental class of $P_{0}$, and let $v \in H^{k}\left(P_{m} ; \boldsymbol{Q}\right)$ where $k \geqq j_{m}$. Then the triple $\left(\odot,\left\{u_{j}\right\}, v\right)$ is the universal example for a higher order cohomology operation $\Phi$ defined as follows. For a CW complex $X$ and classes $x_{j} \in H^{n_{j}}(X ; Q), \Phi\left\{x_{j}\right\}$ is defined and contains $y \in H^{k}(X ; Q)$ if and only if there is a map $g: X$ $\rightarrow P_{m}$ such that $g^{*} u_{j}=x_{j}$ for $j=1, \cdots, s$ and $g^{*} v=y$. If $Y$ is an arbitrary space, let $f: X \rightarrow Y$ be a weak homotopy equivalence from a CW complex $X$ to $Y$. Then we set $\Phi\left\{y_{j}\right\}=f^{*} \Phi\left\{f^{*-1} y_{j}\right\}$ (see $[1, \mathrm{p}$. 54-55]).

Definition 1 . We say that $\Phi$ is a 1 -connected rational cohomology operation (of $s$ variables and of degree $k$ ) if $\Phi$ has a rational universal example $\left(\odot,\left\{u_{j}\right\}, v\right)$ as described above where $\operatorname{dim} u_{j}>1$ for $j=1$, $\cdots, s$, and $\operatorname{dim} v=k$.

LEMMA 2. Let $P$ be a simply connected space whose rational cohomology has finite type and such that $\Omega P$ has the homotopy type of a rational $G E M$. Let $\sigma$ be the loop suspension homomorphism. Then every class in $\operatorname{Im} \sigma$ is a linear combination of the fundamental classes of $\Omega P$.

Proof. $H^{*}(\Omega P ; \mathbf{Q})$ is a commutative, associative Hopf algebra over $Q$. By Lemma 4.17 of [5], the natural map from primitives to indecomposables, $P H^{*}(\Omega P, Q) \rightarrow Q H^{*}(\Omega P ; Q)$, is a monomorphism. As noted in the first paragraph, every class in $H^{*}(\Omega P ; Q)$ is a polynomial on the fundamental classes of $\Omega P$. The lemma now follows since $\operatorname{Im} \sigma \subset P H^{*}(\Omega P ; Q)$.

Lemma 3. Let ( $\left.P,\left\{u_{j}\right\}, v\right)$ be the universal example for a 1-connected rational cohomology operation. Then $\Omega P_{m}$ has the homotopy type of a rational GEM.

Proof. Since $P_{0}$ is a rational GEM, so is $\Omega P_{0}$. Assume, inductively, that $\Omega P_{n} \simeq L \times K\left(Q^{t}, q\right)$ where $Q^{t}$ is $t$-dimensional rational vector space, $q=j_{n+1}-1$, and $L$ is a rational GEM with no factor of degree q. $\Omega P_{n+1}$ is the fiber space induced by the map $\Omega k: \Omega P_{n} \rightarrow K(Q, q)$. Let $z$ be the fundamental class of $K(Q, q)$. By Lemma 2 , since $(\Omega k)^{*}(z)$ $\in \operatorname{Im} \sigma$, there is a map $g: K\left(Q^{t}, q\right) \rightarrow K(Q, q)$ such that $(\Omega k)^{*}(z)$ $=p^{*} g^{*}(z)$, and so $\Omega k \simeq g p$, where $p: \Omega P_{n} \rightarrow K\left(Q^{t}, q\right)$ is the projection. Thus if $E$ is the fiber space induced by $g$, then $\Omega P_{n+1} \simeq L \times E$. It remains to show that $E$ has the homotopy type of a rational GEM.

Clearly the homomorphism $g_{*}: \pi_{q}\left(K\left(Q^{t}, q\right)\right) \rightarrow \pi_{q}(K(Q, q))$ is either 0 or an epimorphism. In the first case $g$ itself is null homotopic, so $E \simeq K\left(Q^{t}, q\right) \times K(Q, q-1)$. In the second case the homotopy long exact sequence for the fibration $K(Q, q-1) \rightarrow E \rightarrow K\left(Q^{t}, q\right)$ implies 
that $\pi_{j}(E)=0$ if $j \neq q$ and $\pi_{q}(E)=Q^{t-1}$ and so $E=K\left(Q^{t-1}, q\right)$.

THEOREM 4. Let $\Phi$ be a 1-connected rational cohomology operation defined on $\left\{x_{j}\right\} \in H^{*}(X ; Q)$. Then $\Phi\left\{x_{j}\right\}=\sum \lambda_{j} x_{j}+\mathcal{U}$, where $\lambda_{j} \in Q$ and $\mathcal{U}$ is a sum of matrix Massey products.

Proof. Let $\left(P,\left\{u_{j}\right\}, v\right)$ be the universal example for $\Phi$. Then by Lemma $3, \Omega P_{m}$ has the weak homotopy type of a rational GEM. By Lemma $2, \sigma v$ is a linear combination of fundamental classes. Since $j_{m} \leq k$, these fundamental classes must come from $\Omega P_{0}$. Thus $\sigma v$ $=\sum \lambda_{j} \sigma u_{j}$.

J. P. May (Corollary 18 [4]) has shown that the kernel of $\sigma$ is generated by matrix Massey products. Since $v-\sum \lambda_{j} u_{j} \in \operatorname{Ker} \sigma$, the theorem follows by naturality.

Note that the entries of the matrices in a matrix Massey product are not assumed to be taken from among the fundamental classes. For example we could define a nontrivial Massey triple product of the form $\left\langle\left\langle u_{1}, u_{2}, u_{3}\right\rangle, u_{4}, u_{5}\right\rangle$.

CoROllaRy 5 . Let $\theta$ be a stable rational cohomology operation (see $\left[1\right.$, p. 64]). If $\theta$ is defined on $\left\{x_{j}\right\}$ in $\widetilde{H}^{*}(X ; Q)$ where $X$ is a connected space, then we can write $\theta\left\{x_{j}\right\}=\sum \lambda_{j} x_{j}$ for some $\lambda_{j} \in Q$.

Proof. Since $\theta$ is stable, there is a 1 -connected rational cohomology operation $\Phi$ such that $s \theta\left\{x_{j}\right\}=\Phi\left\{s x_{j}\right\}$ where $s: H^{n}(X ; Q)$ $\rightarrow H^{n+1}(S X ; Q)$ is the suspension isomorphism. By Theorem 4 , we can write $\Phi\left\{s x_{j}\right\}=\sum \lambda_{j} s x_{j}+\mathcal{u}$. But $\mathcal{u}$ is a sum of matrix Massey products defined in $H^{*}(S X ; Q)$ and therefore, by the dual of Theorem 5 [3], it is identically 0 . Thus $\theta\left\{x_{j}\right\}=\sum \lambda_{j} x_{j}$.

Example 6. Donald W. Kahn [2] has defined a class of secondary cohomology operations with real coefficients which he calls the generalized double and triple products. We shall describe the analogue of these operations in rational cohomology. Let $u \in H^{p}(X ; Q)$ and $v \in H^{q}(X ; Q)$ where $p$ is even, $q$ is odd and $u v=0$. Note that $v^{2}=0$. Then the rational generalized double product $\langle u, v\rangle_{n}(n \geqq 1)$ is defined and has dimension $n(p+q)+q-n$.

The universal example for this operation is $\left(P,\{u, v\}, w_{n}\right)$ where $P$ is induced from the "cup product" pairing $k$

$$
\begin{aligned}
K(\boldsymbol{Q}, p+q-1) & \stackrel{i}{\rightarrow} P \\
& \downarrow \pi \\
K(\boldsymbol{Q}, p) & \times K(\boldsymbol{Q}, q) \stackrel{k}{\rightarrow} K(\boldsymbol{Q}, p+q) .
\end{aligned}
$$

To define $w_{n}$ we examine the Serre spectral sequence of the above fibration. 




In the above diagram, $x$ is the fundamental class of $K(Q, p+q-1)$ and $d_{p+q} x=u v$. For dimension reasons it is clear that $v \otimes x^{n}$ in $E_{2}$ survives to $E_{\infty}$. We call $w_{n}$ the class it represents in $H^{n(p+q)+q-n}(P ; Q)$.

It can be shown that $\langle u, v\rangle_{n} \subset \pm n !\left\langle v, \cdots, v, u^{n}\right\rangle(n+1) v^{\prime} s$. For example let $a$ and $b$ be cocycle representatives of $u$ and $v$ respectively and let $\delta c=a b$. Then setting

$$
a_{1,1}=a_{2,2}=a, \quad a_{3,3}=b, \quad a_{1,2}=\frac{1}{2}\left(a \cup_{1} a\right), \quad a_{2,3}=-c
$$

we have a defining system [3] for $\langle v, v, u\rangle$ with related cocycle $a c-\frac{1}{2}\left(a \cup_{1} a\right) b$. The class of this cocyle in $E_{2}$ is clearly $v \otimes x$.

Thus these secondary operations are actually degenerate higher order Massey products. The generalized triple products can be described in a similar manner.

\section{BIBLIOGRAPHY}

1. J. F. Adams, On the non-existence of elements of Hopf invariant one, Ann. of Math. 72 (1960), 20-104.

2. D. W. Kahn, Secondary cohomology operations which extend the triple product, Pacific J. Math. 13 (1963), 127-139.

3. D. Kraines, Massey higher products, Trans. Amer. Math. Soc. 124 (1966), 431449.

4. J. P. May, The algebraic Eilenberg-Moore spectral sequence, (to appear).

5. J. Milnor and J. C. Moore, On the structure of Hopf algebras, Ann. of Math. 81 (1965), 211-264.

Haverford College 